\title{
Pedalear sin fatigarse: análisis de infraestructura ciclística urbana basado en la energía del pedaleo*
}

\author{
Daniel Orellana \\ Ivanna Martínez-Pesántez \\ Paúl Pulla-Tenemaza \\ Andrés Tapia-Sisalima \\ Universidad de Cuenca (Ecuador). LlactaLAB - Ciudades Sustentables. \\ Departamento Interdisciplinario de Espacio y Población \\ daniel.orellana@ucuenca.edu.ec \\ ivanna.martinezp@ucuenca.ec \\ esteban.pulla94@ucuenca.ec \\ andres.tapia@ucuenca.ec
}

\section{Resumen}

Una de las estrategias para la promoción de la bicicleta como medio de transporte urbano es el diseño y la implementación de la infraestructura necesaria. Ello incluye ciclovías, estacionamientos de bicicletas y sistemas de préstamo de los vehículos. La ubicación espacial de estas infraestructuras es un aspecto clave para garantizar su éxito. En este artículo proponemos la ampliación de un método de análisis de infraestructura ciclística basado en el esfuerzo del pedaleo que toma en cuenta la velocidad, la pendiente del terreno, las intersecciones y la superficie de rodadura para calcular la energía requerida para el desplazamiento. Además, presentamos una herramienta de análisis en sistemas de información geográfica que permite, a partir de datos libres, modelar el nivel de servicio de las estaciones de préstamo de bicicletas, determinar las rutas óptimas para conformar una red de ciclovías y optimizar la ubicación de las estaciones de préstamo utilizando análisis de redes y algoritmos de localización-asignación. Los resultados demuestran la viabilidad del método para apoyar la toma de decisiones sobre infraestructura ciclística urbana.

Palabras clave: movilidad activa; infraestructura ciclística; SIG; movilidad sostenible

* Este estudio es parte del proyecto de investigación Estudio de los patrones de movilidad de ciclistas y peatones para una movilidad sustentable, ganador del XIII concurso de proyectos de investigación financiado por la Dirección de Investigaciones de la Universidad de Cuenca. Los autores agradecen a Elizabeth Rodas, Adriana Quezada y Danilo Mejía sus valiosos comentarios y sugerencias para este manuscrito. 
Resum. Pedalejar sense fatigar-se: anàlisi d'infraestructura ciclista urbana basada en l'energia de pedaleig

Una de les estratègies per promoure la bicicleta com a mitjà de transport urbà és el disseny i la implementació de la infraestructura adient. Això inclou carril bici, estacionaments de bicicletes i sistemes de préstec dels vehicles. La ubicació espacial d'aquestes infraestructures és un aspecte clau per garantir-ne l'èxit. En aquest article proposem l'ampliació d'un mètode d'anàlisi d'infraestructura ciclista que té en compte la velocitat, el pendent del terreny, les interseccions i la superfície de rodament per calcular l'energia requerida per al pedaleig. A més, presentem una eina SIG que permet, a partir de dades lliures, modelar el nivell de servei de les estacions de préstec de bicicletes, determinar les rutes òptimes per conformar una xarxa de carril bici i optimitzar la ubicació de les estacions de préstec utilitzant anàlisi de xarxes i algorismes de localització-assignació. Els resultats demostren la viabilitat del mètode per donar suport a la presa de decisions sobre infraestructura ciclista urbana.

Paraules clau: mobilitat activa; infraestructura ciclista; SIG; mobilitat sostenible

Résumé. Pédaler sans se fatiguer: Analyse de l'infrastructure cyclable urbaine basée sur l'énergie de pédalage

L'une des stratégies de promotion du vélo comme moyen de transport urbain est la conception et la mise en œuvre des infrastructures, y compris les pistes cyclables, le stationnement des bicyclettes et les systèmes de partage de bicyclettes. La localisation spatiale de ces infrastructures est un élément clé pour garantir leur succès. Dans cet article, nous proposons l'élargissement d'une méthode d'analyse de l'infrastructure cyclable qui tient compte de la vitesse, de l'inclinaison, des intersections et de la surface de roulement pour estimer l'énergie nécessaire au pédalage. Nous présentons également un outil de système d'information géographique qui permet de modéliser le niveau de service des stations de vélos, de déterminer les itinéraires optimaux pour former un réseau de pistes cyclables et d'optimiser la localisation des stations de prêt en utilisant des algorithmes d'analyse et de localisation réseau. Les résultats démontrent la viabilité de la méthode pour soutenir la prise de décision sur l'infrastructure des bicyclettes urbaines.

Mots-clés: mobilité active; infrastructure de vélo; SIG; mobilité durable

Abstract. Pedal without fatigue: Analysis of urban cycling infrastructure based on pedalling energy

An effective strategy for the promotion of cycling as urban transport is the design and implementation of cycling infrastructure, such as cycle paths, bicycle parking and bicyclesharing schemes. The spatial location of these infrastructures is a key aspect to guarantee their success. In this contribution, we propose the extension of a method of analysis that takes into account the speed, slope, intersections and rolling surface to estimate the energy required for pedalling. In addition, we present a GIS-based assignment tool that enables modelling the service level of bicycle loan stations, optimising their location and determining the optimal routes to design a cycle path network using network analysis and localization algorithms with open data. The results demonstrate the viability of the method to support decision-making on urban cycling infrastructure.

Keywords: active mobility; cycling infrastructure; GIS; sustainable mobility 


\section{Sumario}
1. Introducción
4. Resultados
2. Métodos
5. Conclusiones
Referencias bibliográficas

3. Aplicación: análisis de

\section{Introducción}

La priorización de la movilidad a pie y en bicicleta, junto con la mejora de los sistemas de transporte público, han demostrado ser estrategias eficaces para mejorar la movilidad urbana. Específicamente, la promoción de la bicicleta como medio de transporte ayuda de manera fundamental a conseguir los cuatro objetivos principales del espacio público propuestos por Gehl: vitalidad, seguridad, sostenibilidad y salubridad (Gehl, 2014). A su vez, la movilidad en bicicleta es económica para las ciudades y las personas, ya que las inversiones en adquisición, infraestructura y mantenimiento, así como los costos ambientales y sociales, son mucho menores que aquellos del transporte motorizado (Buehler y Pucher, 2012), cuyas externalidades tendrían un costo del 18\% del ingreso promedio en ciudades latinoamericanas (Hidalgo y Huizenga, 2013). Por otro lado, la movilidad en bicicleta es socialmente equitativa, ya que aumenta las opciones de movilidad autónoma para varios grupos sociales, tales como menores de edad y personas con movilidad en silla de ruedas que también se benefician de la infraestructura ciclística (De Nazelle et al., 2011).

Son varios los factores que influyen en la decisión de una persona de adoptar un medio de transporte determinado, y estos tienen una importancia especial en el caso de la bicicleta (Hanson, 2004). Entre estos factores están las condiciones del terreno, la superficie de las carreteras, el clima, el tipo de ruta, la distancia, el tiempo, la economía y las facilidades de movilidad (existencia de ciclovías, señalización, pendientes del terreno), entre otros (Iseki y Tingstrom, 2014). Entre las estrategias más eficaces para aumentar el número de viajes en bicicleta se encuentran el mejoramiento y la creación de infraestructura ciclística urbana, incluyendo la construcción de redes de ciclovías y la implementación de sistemas de bicicleta pública, tal como lo demuestran las experiencias en ciudades como Montreal, Washington D.C., Nueva York, Chicago, Bogotá y Medellín, entre muchas otras (Fishman et al., 2013; Greenstein, 2015; Hidalgo y Huizenga, 2013; Lugo, 2013; Sener et al., 2009; Shaheen et al., 2010; Torres et al., 2013). El éxito de estos sistemas provee evidencias sobre los potenciales impactos positivos en la reducción del tráfico $\mathrm{y}$, consecuentemente, del ruido y la contaminación, así como en la salud física y mental de las personas (Fraser y Lock, 2011).

La ubicación de la infraestructura ciclística es determinante en su éxito y efectividad (Larsen et al., 2013). Esto es especialmente cierto en el caso de las estaciones de sistemas de bicicleta pública, pues los usuarios deberán poder 
tomar una bicicleta cerca de su punto de origen y dejarla cerca de su punto de destino. Es por esto que, en muchos casos, estos servicios públicos buscan analizar la cobertura de las estaciones atendiendo a un criterio de distancia euclidiana o, en el mejor de los casos, una distancia en la red vial (Institute for Transportation and Development Policy, 2013). Este tipo de análisis es frecuentemente implementado en sistemas de información geográfica (SIG) como un búfer euclidiano o un área de servicio en análisis de redes (Olaya, 2014). De la misma manera, el trazado de redes de ciclovías debe tomar en cuenta el contexto urbano en términos de cercanía, conectividad, seguridad y topografía, entre otros.

El análisis espacial con sistemas de información geográfica (SIG) es un método eficaz para el análisis y la planificación de la ubicación de infraestructura ciclística. Por ejemplo, Yamashita et al. (1999) han demostrado el uso de SIG y de imágenes satélite para la planificación de redes de ciclovías. De Baets et al. (2011) desarrollaron un método basado en SIG para identificar cuellos de botella en el sistema de ciclovías tomando en consideración la topografía, el ancho de los carriles y la presencia de rompevelocidades. Larsen et al. (2013) desarrollaron un índice de priorización para la ubicación de infraestructura ciclística a partir de los viajes actuales y de los potenciales, la prioridad asignada por ciclistas a los segmentos de calle y la ubicación de accidentes que involucraron a ciclistas.

La mayoría de metodologías para el análisis de infraestructura ciclística han sido implementadas a partir de enfoques de accesibilidad similares a aquellos que sirven para modos tradicionales de transporte, muchos de los cuales asumen la optimización de la distancia o del tiempo el momento de realizar un viaje y decidir una ruta óptima (Suxia Liu y Xuan Zhu, 2004). Sin embargo, la movilidad en bicicleta tiene particularidades importantes, pues su usuario deberá invertir una cantidad de su propia energía para transportarse, la cual dependerá de la velocidad de desplazamiento, de la pendiente, del peso combinado de la persona y de la bicicleta, y de la fricción entre la rueda y la superficie, entre otros condicionantes. De esta manera, para realizar un viaje utilitario en bicicleta, el usuario buscará optimizar el esfuerzo de su pedaleo, lo cual será determinante para seleccionar la ruta a seguir e incluso para la decisión de hacer dicho viaje en bicicleta u otro medio de transporte (Iseki y Tingstrom, 2014). Es necesario, por lo tanto, modelar el esfuerzo necesario para movilizarse en bicicleta, de manera que este factor pueda ser utilizado para la planificación de infraestructura ciclística.

Iseki y Tingstrom (2014) describen un método basado en análisis de redes para calcular el área de influencia de un punto de infraestructura ciclística con relación a un punto utilizando la energía como valor de impedancia. Los autores utilizaron la pendiente de los segmentos de calle y las intersecciones que los conectan para estimar la cantidad de energía consumida al movilizarse en bicicleta. De esta manera pudieron determinar, a través de un tipo particular de análisis de redes (área de servicio), las zonas de influencia que se podrían abarcar en bicicleta a partir de un punto específico con una cantidad 
de energía determinada. En dicho trabajo se utiliza el término cuenca ciclable (en inglés, bikesheed) como una analogía con el término cuenca hídrica (en inglés, watershed).

El objetivo del presente artículo es ampliar la metodología y la aplicación del análisis de movilidad en bicicleta basado en energía propuesto por Iseki y Tingstrom (2014) y demostrar su viabilidad para analizar diferentes aspectos de la infraestructura ciclística urbana: modelar las áreas de influencia y el nivel de servicio de las estaciones del sistema de bicicleta pública, determinar las rutas óptimas que conforman la red de ciclovías de conectividad de las estaciones y optimizar la ubicación de las estaciones utilizando algoritmos de localizaciónasignación. Esta investigación representa avances con respecto a la propuesta original en varios aspectos: por una parte, se incorpora en el modelo el efecto que los diferentes tipos de superficie de rodadura tiene en el consumo de energía utilizando coeficientes publicados previamente (Hölzel et al., 2012). Por otro lado, se utilizan datos abiertos de topografía y de red vial disponibles para la mayoría de zonas urbanas del planeta a través de la plataforma OpenStreetMap (2016) o de las administraciones locales, lo que garantiza la aplicabilidad y la replicabilidad a nivel global. Además, se presenta una caja de herramientas que implementa los geoprocesos necesarios en un sistema de información geográfica, de manera que pueda ser fácilmente replicable en otras ciudades. Finalmente, se demuestra la aplicación del método no solamente para el análisis de áreas de influencia, sino también para el diseño de redes de ciclovías y la optimización de estaciones de préstamo de bicicletas.

El enfoque planteado fue aplicado para el análisis de distribución de estaciones y ciclovías potenciales del futuro sistema de bicicletas públicas propuesto por el gobierno local de la ciudad de Cuenca (Ecuador). Los resultados permitirán ayudar a tomar decisiones en las etapas de planificación y operación de nueva infraestructura ciclística en esta y otras ciudades, así como evaluar la infraestructura existente.

Las siguientes secciones del presente artículo se estructuran como sigue: la sección 2 detalla la metodología implementada para el análisis de cuencas ciclables basada en el esfuerzo del pedaleo, haciendo énfasis en las diferencias con respecto al método original. La sección 3 presenta el caso de estudio de análisis de la nueva infraestructura ciclística propuesta en la ciudad de Cuenca (Ecuador). La sección 4 presenta los principales resultados obtenidos para el caso de estudio. Finalmente, la sección 5 menciona las principales conclusiones, así como las limitaciones de este estudio y los siguientes pasos de nuestra investigación.

\section{Métodos}

En esta sección se presenta una ampliación del método de análisis de movilidad en bicicleta basado en energía, de Iseki y Tingstrom (2014). En primer lugar, se explicita el modelo conceptual en el que se basa el método; a continuación, se detalla la parametrización del modelo y los valores específicos utilizados 
en el presente estudio, y, finalmente, se explica la implementación del modelo en un sistema de información geográfica.

\subsection{Modelo conceptual}

El modelo conceptual de energía por pedaleo propuesto por Iseki y Tingstrom (2014) se basa en los siguientes supuestos:

a) $\mathrm{Al}$ momento de transportarse en bicicleta, un ciclista buscará minimizar el esfuerzo que realiza al pedalear.

b) El esfuerzo del pedaleo puede ser modelado como la potencia necesaria para mover la masa del ciclista y la bicicleta a una velocidad constante.

c) Es posible asignar a cada segmento de calle un valor de energía necesaria para recorrerlo en función de la potencia y el tiempo.

d) Al llegar a una intersección, el ciclista debe continuar o detenerse según la prioridad de circulación de los segmentos conectados a la intersección, y este comportamiento puede implicar un consumo adicional de energía, al tener que detenerse y acelerar nuevamente hasta alcanzar la velocidad nominal. La energía consumida dependerá del tipo de intersección.

e) La energía necesaria para recorrer la red vial puede ser entendida como un factor de impedancia y, por lo tanto, es posible utilizar algoritmos de análisis de redes utilizando este valor para calcular cuencas ciclables y rutas óptimas.

A partir de esta serie de supuestos, es posible crear un grafo de red en el que los vértices representan los ejes de calle y los nodos representan las intersecciones, y así asignar como valores de impedancia la energía consumida por el ciclista en cada uno de los segmentos y las intersecciones. Para estimar la energía es necesario, en primer lugar, obtener la potencia en vatios necesaria para mover la masa del ciclista y la bicicleta a una velocidad constante. Para ello se utiliza la ecuación propuesta por Wilson y Papadopoulos (2004) (ecuación 1), que toma en cuenta la resistencia aerodinámica, la resistencia a la pendiente y la resistencia a la rodadura

$$
P=\left[K_{A} \cdot\left(V+V_{W}\right)^{2}+m \cdot g \cdot\left(s+C_{R}\right)\right] \cdot V
$$

donde $P$ es la potencia en vatios (julios por segundo) $K_{A}$ es el factor de arrastre, $V$ es la velocidad de desplazamiento del ciclista en $\mathrm{ms}^{-1}, V_{W}$ es la velocidad del viento en $\mathrm{ms}^{-1}$, $m$ es la masa conjunta del ciclista y la bicicleta en kilogramos, $g$ es la aceleración de la gravedad $\left(\mathrm{ms}^{-2}\right), s$ es la pendiente relativa y, finalmente, $C_{R}$ es el coeficiente de rodadura producido por la fricción entre la rueda de la bicicleta y la superficie del suelo.

Una vez obtenida la potencia, es posible calcular la energía necesaria $E$ para recorrer un segmento de calle utilizando la ecuación 2

$$
E=P l V^{-1}
$$


donde $E$ es la energía consumida por el ciclista para recorrer un segmento de longitud $l$ a una velocidad $V$ utilizando una potencia $P$.

\subsection{Parametrización del modelo}

Algunos parámetros del modelo, tales como $m$ o $V w$ y $C_{R}$, pueden ser altamente variables. Para esta investigación, se han utilizado los valores sugeridos por Iseki y Tingstrom (2014) (tabla 1). Sin embargo, como se verá más adelante, la implementación desarrollada es completamente parametrizable y estos valores pueden ser actualizados de forma dinámica durante el análisis.

La pendiente $s$ se calcula como el cociente entre la diferencia de altura entre el inicio y el final de un segmento de calle y su longitud. Los valores de altura se obtienen mediante un análisis de superposición entre los puntos inicial y final de cada segmento y un modelo digital de elevaciones (MDE). En segmentos largos es probable que la pendiente no sea uniforme, por lo tanto, estos se han segmentado en longitudes no mayores a cien metros (Winters et al., 2010).

En el trabajo original de Iseki y Tingstrom, se utilizó un valor constante en el coeficiente de rodadura $C_{R}=0.004$ para todos los segmentos de calle. En esta investigación buscamos mejorar la precisión del modelo asignando valores de $C_{R}$ a cada segmento en función del tipo de superficie de rodadura, según los valores propuestos en Hölzel et al. (2012) (tabla 2).

En el caso de la energía consumida en las intersecciones, se asumen valores en función del tipo de giro que debe realizar el ciclista y el tipo de vías que confluyen en las intersecciones clasificadas como locales (calles de menor jerarquía) o arteriales (avenidas de mayor jerarquía). Los giros más costosos en términos

Tabla 1. Valores asignados a los parámetros del modelo

\begin{tabular}{cc}
\hline Parámetro & Valor \\
\hline$K_{A}$ & 0.245 \\
$V$ & $4 \mathrm{~ms}^{-1}$ \\
$V W$ & $0 \mathrm{~ms}^{-1}$ \\
$m$ & $80 \mathrm{~kg}$ \\
$g$ & $9.807 \mathrm{~ms}^{-2}$ \\
\hline
\end{tabular}

Fuente: Iseki y Tingstrom (2014).

Tabla 2. Coeficientes de rodadura para diferentes tipos de superficie

\begin{tabular}{lc}
\hline Tipo de superficie de rodadura & $C_{R}$ \\
\hline Asfalto nuevo & 0.0071804 \\
Hormigón nuevo & 0.0063459 \\
Adoquín & 0.0103079 \\
Asfalto desgastado & 0.0089477 \\
Hormigón desgastado & 0.0066998 \\
Gravilla & 0.0132250 \\
\hline
\end{tabular}

Fuente: Hölzel et al. (2012). 
Tabla 3. Energía consumida en las intersecciones

\begin{tabular}{llllccc}
\hline Tipo de giro & Desde & A través & Hacia & Paradas & $\begin{array}{c}\text { Energía } \\
\text { consumida (kJ) }\end{array}$ & $\begin{array}{c}\text { Distancia } \\
\text { equivalente (m) }\end{array}$ \\
\hline Giro hacia & Local & Arterial & Arterial & 2 & 1.466 & 208 \\
la izquierda & Arterial & Arterial & Arterial & 2 & 1.466 & 208 \\
& Arterial & Local & Local & 1 & 0.733 & 104 \\
& Local & Local & Local & 1 & 0.733 & 104 \\
\hline Giro hacia & Local & Local & Local & 0.5 & 0.367 & 52 \\
la derecha & Local & Arterial & Arterial & 1 & 0.733 & 104 \\
& Arterial & Arterial & Arterial & 1 & 0.733 & 104 \\
& Arterial & Local & Local & 0 & 0 & - \\
\hline Adelante & Local & Local & Local & 0.5 & 0.367 & 52 \\
& Local & Arterial & Local & 1 & 0.733 & 104 \\
& Arterial & Arterial & Arterial & 1 & 0.733 & 104 \\
& Arterial & Local & Arterial & 0 & 0 & - \\
\hline
\end{tabular}

Fuente: Iseki y Tingstrom (2014).

de energía son aquellos en los que el ciclista debe detenerse completamente por dos ocasiones en la intersección antes de realizar el giro y volver a acelerar a la velocidad nominal, como es el caso de un giro hacia la izquierda hacia una vía arterial. Esto requiere un consumo de energía de $1.466 \mathrm{~kJ}$, equivalente a recorrer 208 metros en terreno plano. En otros casos, el consumo de energía en la intersección es menor, ya que solo se requiere detenerse en una ocasión o disminuir la velocidad. Los valores para los diferentes tipos de giro se presentan en la tabla 3.

Finalmente, es necesario modelar los factores restrictivos o preferenciales de circulación en la red vial, tales como el sentido de circulación, la preferencia por ciclovías segregadas o la prohibición de cruzar segmentos o intersecciones determinadas. Estos factores se modelan como ponderadores de los segmentos en función de los atributos de la red y pueden ser asignados de forma dinámica durante el análisis de cada caso específico.

\subsection{Generación de una red vial topológica con valores de energía}

A partir del modelo parametrizado con los valores indicados, se construyó una red vial topológica utilizando el sistema de información geográfica ArcGIS 10.3 con las extensiones Spatial Analyst y Network Analyst (ESRI, 2015). En primer lugar, se obtuvo la red vial de la zona de estudio (área urbana de Cuenca, Ecuador) desde la plataforma OpenStreetMap (OpenStreetMap, 2016) y se realizó un proceso de verificación y depuración. Con los datos depurados se construyó una red con topología completa (con las relaciones entre ejes viales e intersecciones representadas de forma explícita) utilizando el complemento OSM Editor para ArcGIS (Hanson y Seeger, 2014).

En segundo lugar, se obtuvieron los valores de elevación en los extremos de cada segmento de calle utilizando un modelo digital de elevaciones con una reso- 
lución espacial de 3 metros obtenido del proyecto SIGTierras (MAGAP, 2012) y se calcularon los valores de pendiente de cada segmento. Ya que cada segmento puede ser, en principio, recorrido en ambos sentidos, los valores de pendiente se almacenaron en dos nuevos atributos con el mismo valor y con signo opuesto.

A continuación, se elaboraron tablas de correspondencia entre los atributos de cada segmento de calle definidos en OpenStreetMap y los valores necesarios para el cálculo de la potencia. Específicamente, se utilizaron los atributos almacenados en las etiquetas superficie de rodadura $\left(\right.$ surface $\left.={ }^{*}\right)$ y tipo de vía $\left(\right.$ highway $={ }^{*}$ ). Las tablas de correspondencia fueron unidas a las tablas de atributos de los segmentos y de las intersecciones y se calcularon los valores de energía consumida en cada segmento y en cada intersección según las ecuaciones presentadas anteriormente. A cada segmento se le asignaron dos valores de energía, uno para cada sentido de recorrido. Los valores negativos fueron reasignados con 0 , ya que representan segmentos donde el ciclista no necesita pedalear debido a la pendiente descendente. Por último, se recalculó la red topológica utilizando como valor de impedancia la energía $E$.

Todos los pasos han sido implementados como un proceso automatizado en el sistema de información geográfica ArcGIS utilizando el entorno Model Builder (ESRI, 2015). Los archivos correspondientes se encuentran disponibles de forma libre en <http://llactalab.ucuenca.edu.ec> junto con los archivos en formato XML, que permiten generar los valores de energía para cada segmento de una red vial de OpenStreetMap. De esta manera es posible replicar el estudio de forma automática para cualquier ciudad o región donde exista esta información.

\section{Aplicación: análisis de infraestructura ciclística}

Para demostrar la viabilidad y la utilidad del método planteado, se ha analizado como caso de estudio el sistema de bicicleta pública propuesto por el gobierno municipal de Cuenca (Ecuador), en el cual se propone establecer veinte estaciones de préstamo distribuidas en la zona central de la ciudad.

El estudio consistió en:

a) Calcular las áreas de influencia (cuencas ciclables) y el nivel de servicio del sistema.

b) Determinar la red vial que conectará las estaciones entre sí.

c) Determinar la ubicación óptima de las diez primeras estaciones que se construirán en la primera fase de operación del sistema.

Los análisis se realizaron en el software ArcGIS 10.3 con la extensión Network Analyst.

El área de estudio consiste en la zona urbana de Cuenca (Ecuador). La ciudad, con una población urbana de aproximadamente 352.766 habitantes en el año 2010 (INEC, 2010), presenta una topografía caracterizada por diferentes "plataformas», con pendientes leves de aproximadamente $5 \%$ en dirección 
Figura 1. El área de estudio comprende la zona urbana de la ciudad de Cuenca (Ecuador), caracterizada por una topografía variable y accidentada. Los puntos indican las estaciones del sistema de bicicleta pública propuesto por el gobierno municipal
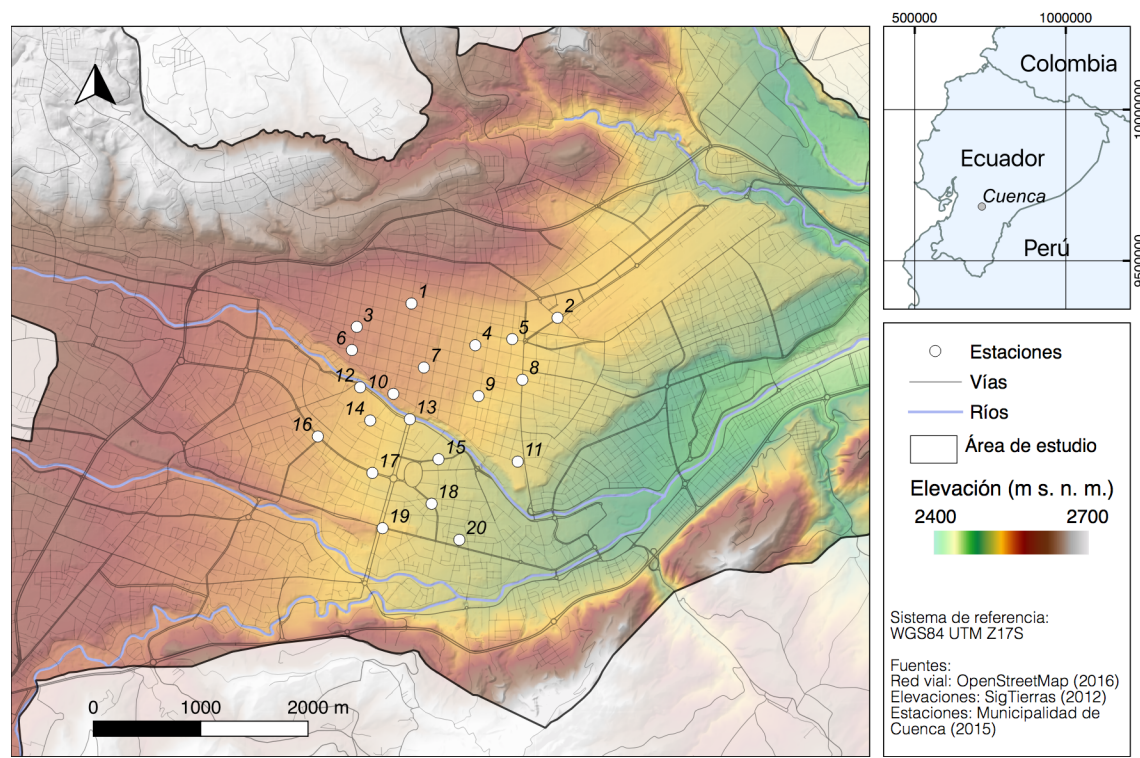

Fuente: elaboración propia a partir de datos de OpenStreetMap (2016), MAGAP (2012) y Municipalidad de Cuenca (2015).

este-oeste y desniveles fuertes entre la zona central y sur, así como un relieve accidentado en las áreas periféricas. La figura 1 muestra un mapa de la zona de estudio con la ubicación de las estaciones del sistema propuesto.

Para modelar cada cuenca ciclable, es decir, el área de influencia de cada estación del sistema de bicicleta pública, se calcularon en primer lugar dos polígonos de áreas de servicio (service area). El primer polígono representa la zona a la que se puede acceder en bicicleta desde la estación de préstamo (llamada «desde») con un consumo máximo de energía de $50 \mathrm{~kJ}$, mientras que el segundo polígono representa la zona desde la que se puede llegar hasta dicha estación (por lo que se le ha llamado «hacia»). A continuación, se calculó la intersección geométrica entre los dos polígonos para representar el área efectiva de influencia (figura 2), superficie en la cual todos los viajes desde y hacia la estación analizada consumirán un máximo de $50 \mathrm{~kJ}$ en total. Finalmente, se calculó la cobertura del sistema agregando las cuencas ciclables de todas las estaciones y se analizó el número de aquellas que cubren cada parte de la zona de estudio para estimar el nivel de servicio.

El segundo análisis consistió en determinar los segmentos de calle donde se implementarán las ciclovías que conectarán las estaciones de préstamo. Para esto, se realizó un análisis de ruta óptima entre las estaciones de préstamo y los 
Figura 2. Ejemplo del área efectiva de influencia de una estación de préstamo de bicicletas con un consumo máximo de $50 \mathrm{~kJ}$ de energía

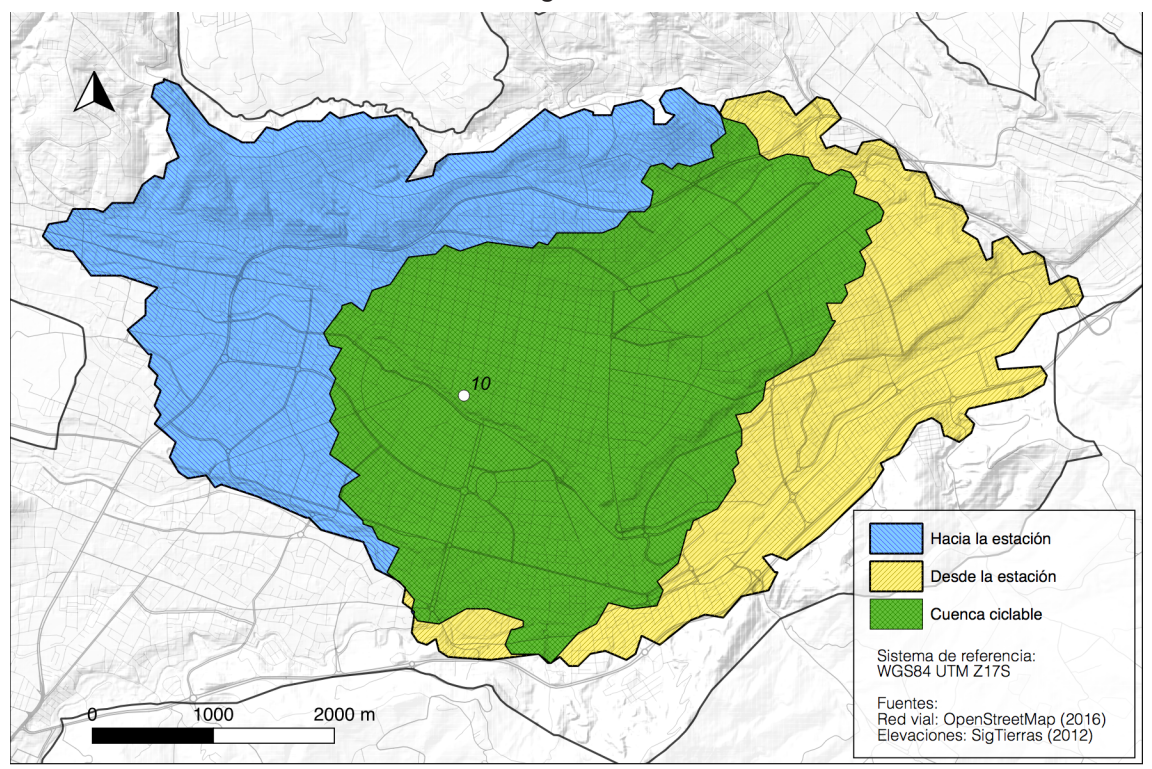

Fuente: elaboración propia a partir de datos de OpenStreetMap (2016) y MAGAP (2012).

equipamientos y servicios existentes en el área, siempre utilizando la energía como atributo de impedancia. Una vez determinadas todas las rutas, se realizó un conteo del número de rutas que atraviesan cada segmento de calle, y el resultado se utilizó como guía para determinar la red de ciclovías, priorizando los segmentos de calle con mayor número de rutas. Adicionalmente, se calculó la energía necesaria para pedalear entre cada par de estaciones del sistema. Los resultados fueron consignados en una matriz de origen y destino.

El último análisis consistió en optimizar la ubicación de las estaciones para maximizar su cobertura. La implementación del sistema de bicicleta pública tiene previsto iniciar con diez estaciones durante el primer año y en una segunda fase implementar diez estaciones más para completar las veinte previstas. La selección de las estaciones en la primera fase debe garantizar la mayor cobertura posible de destinos, para, en la segunda fase, densificar el número de estaciones. La optimización se realizó utilizando un algoritmo de localización-asignación con una función objetivo de maximización de asistencia (ESRI, 2015), seleccionando las diez ubicaciones que maximicen el número de destinos (negocios y servicios) dentro del área de influencia de 50 kilojulios. Cada equipamiento o servicio recibió un peso relativo en el algoritmo en función de la frecuencia esperada de uso (diaria, semanal, mensual, etc.). La ubicación de los equipamientos y de los servicios fue obtenida a partir de la información de usos del suelo del municipio de Cuenca (Ecuador). 


\section{Resultados}

\subsection{Cuencas ciclables}

El área de influencia del sistema de bicicleta pública propuesto en Cuenca, tomando en cuenta un consumo máximo de energía de $50 \mathrm{~kJ}$, cubre una superficie total de 2238 ha, que representan un 31,7\% del área urbana de la ciudad. La distribución propuesta de las estaciones implica un nivel de servicio diferenciado, en el que el centro histórico y gran parte de la zona de El Ejido contarían con una cobertura de más de 16 estaciones, mientras que las zonas más periféricas contarían con un servicio de 4 estaciones o menos (figura 3).

La topografía juega un papel importante en la cobertura de las estaciones, pues las áreas con mayores pendientes disminuyen considerablemente la cobertura. Así mismo, las grandes avenidas diseñadas para el transporte motorizado representan una barrera para la accesibilidad en bicicleta debido al esfuerzo que implica cruzarlas, lo que limita la cobertura del servicio.

La tabla 4 detalla la superficie del área de influencia de cada estación y, con fines comparativos, las superficies para áreas de influencia obtenidas mediante la propuesta original de Iseki y Tingstrom y el método convencional basado en el cálculo de distancia de red. Es posible observar que las cuencas ciclables calculadas con la propuesta original sobreestiman en promedio un $24 \%$

Figura 3. Cobertura potencial del sistema de estaciones de bicicleta pública con un límite de energía de $50 \mathrm{~kJ}$. Los colores indican el nivel de servicio (número de estaciones desde las que se puede acceder)

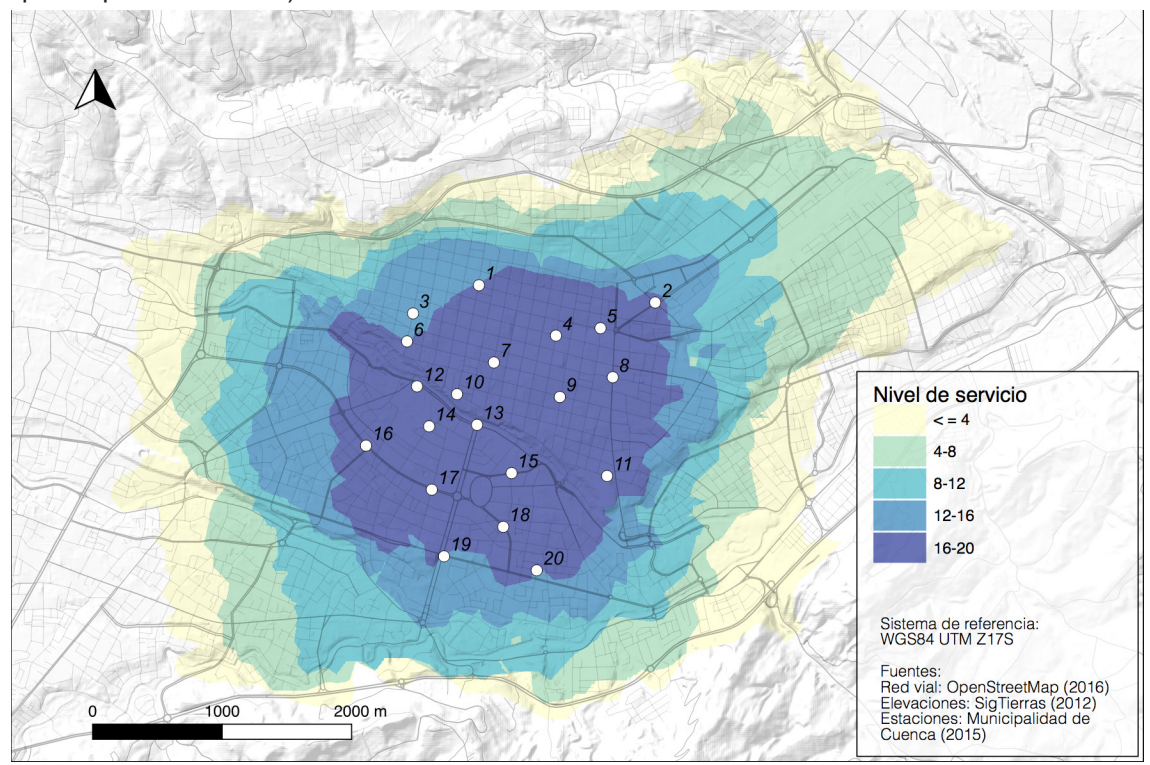

Fuente: elaboración propia a partir de datos de OpenStreetMap (2016), MAGAP (2012) y Municipalidad de Cuenca (2015). 
Tabla 4. Superficie de las cuencas ciclables del sistema de estaciones de bicicleta pública. Entre paréntesis se reporta la diferencia porcentual

\begin{tabular}{|c|c|c|c|c|c|}
\hline \multirow[b]{2}{*}{ Estación } & \multirow{2}{*}{$\begin{array}{c}\text { Superficie de la cuenca } \\
\text { ciclable }\left[\mathrm{km}^{2}\right]\end{array}$} & \multicolumn{2}{|c|}{$\begin{array}{l}\text { Superficie según la } \\
\text { propuesta original de } \\
\text { Iseki y Tingstrom }\end{array}$} & \multicolumn{2}{|c|}{$\begin{array}{l}\text { Superficie según el } \\
\text { método convencional }\end{array}$} \\
\hline & & $\mathrm{km}^{2}$ & diferencia \% & $\mathrm{km}^{2}$ & diferencia \% \\
\hline 1 & 8.98 & 11.75 & $(30.8 \%)$ & 86.88 & (867.5\%) \\
\hline 2 & 10.33 & 13.15 & (27.3\%) & 84.86 & $(721.5 \%)$ \\
\hline 3 & 8.45 & 11.04 & $(30.7 \%)$ & 88.00 & (941.4\%) \\
\hline 4 & 10.64 & 13.45 & (26.4\%) & 84.94 & (698.3\%) \\
\hline 5 & 11.13 & 13.39 & (20.3\%) & 85.79 & $(670.8 \%)$ \\
\hline 6 & 8.71 & 11.12 & $(27.7 \%)$ & 86.87 & (897.4\%) \\
\hline 7 & 10.47 & 13.25 & $(26.6 \%)$ & 84.88 & (710.7\%) \\
\hline 8 & 11.56 & 13.87 & (20.0\%) & 86.22 & $(645.8 \%)$ \\
\hline 9 & 10.22 & 12.95 & (26.7\%) & 84.97 & (731.4\%) \\
\hline 10 & 10.71 & 13.6 & $(27.0 \%)$ & 85.42 & (697.6\%) \\
\hline 11 & 11.68 & 13.86 & $(18.7 \%)$ & 80.90 & (592.6\%) \\
\hline 12 & 10.87 & 13.03 & (19.9\%) & 84.85 & (680.6\%) \\
\hline 13 & 11.00 & 13.46 & $(22.4 \%)$ & 84.07 & (664.3\%) \\
\hline 14 & 10.71 & 12.78 & $(19.3 \%)$ & 81.42 & $(660.2 \%)$ \\
\hline 15 & 10.32 & 12.96 & $(25.6 \%)$ & 80.73 & (682.3\%) \\
\hline 16 & 10.92 & 12.76 & $(16.8 \%)$ & 79.84 & (631.1\%) \\
\hline 17 & 10.78 & 13.06 & $(21.2 \%)$ & 79.93 & (641.5\%) \\
\hline 18 & 9.91 & 12.52 & (26.3\%) & 79.90 & (706.3\%) \\
\hline 19 & 10.22 & 12.55 & (22.8\%) & 79.14 & (674.4\%) \\
\hline 20 & 9.05 & 11.14 & (23.1\%) & 76.11 & (741.0\%) \\
\hline Media & 10.33 & 12.78 & (24.0\%) & 83.29 & $(712.8 \%)$ \\
\hline
\end{tabular}

* Con un valor fijo para el coeficiente de rodadura $\mathrm{Cr}=0.004$.

** Equivalente a una distancia de red de $7.08 \mathrm{~km}$ en terreno plano.

Fuente: elaboración propia.

la superficie en comparación con el método propuesto en este trabajo. Así mismo, los valores obtenidos con el método convencional son en promedio siete veces mayores que aquellos resultantes del método basado en energía. Estos resultados resaltan la importancia de tomar en cuenta la topografía y el tipo de superficie de las vías en el momento de estimar las áreas de influencia de la infraestructura ciclística.

\subsection{Determinación de la red ciclable}

Se generaron un total de 8023 rutas entre las estaciones de préstamo de bicicletas y los equipamientos y servicios existentes en el área. Los segmentos de la red vial por los que recorren las rutas óptimas cubren la mayor parte de vías en la zona central y sur de la ciudad, extendiéndose hacia las afueras (figura 4). Sin embargo, por razones económicas y urbanísticas, no es viable implementar 
Figura 4. Intensidad de uso potencial de los segmentos de la red vial para la conectividad de las estaciones de préstamo con equipamientos y servicios

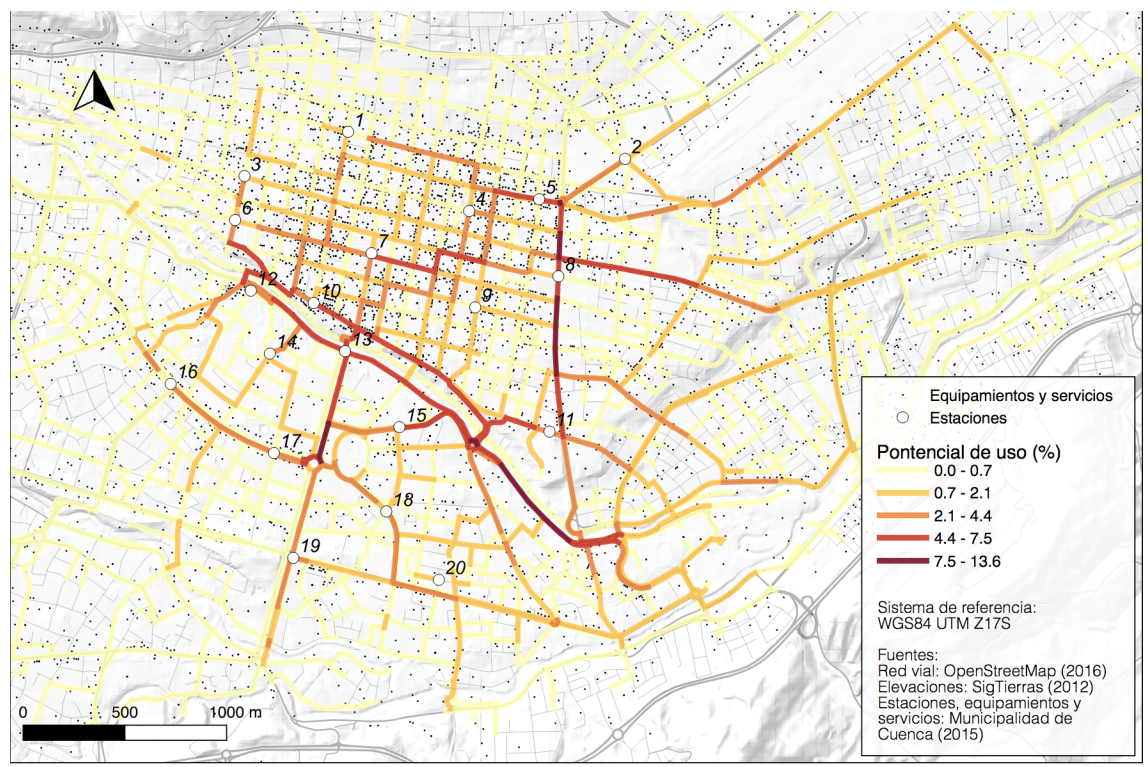

Fuente: elaboración propia con datos de OpenStreetMap (2016), MAGAP (2012) y Municipalidad de Cuenca (2015).

ciclovías en todas las calles, por lo que se debe dar preferencia a los ejes en los cuales se establecerá la red. Se priorizaron, por lo tanto, los segmentos con mayor potencial de uso según las rutas óptimas basadas en energía, y se utilizó este criterio junto con la continuidad espacial y los sentidos de circulación de tráfico para proponer el trazado de la red. La propuesta se presenta en la figura 5. Es importante notar que los valores de las rutas óptimas de energía son útiles para guiar el diseño, pero no son definitorios, ya que es necesario considerar la infraestructura existente, la intensidad del tráfico vehicular y las secciones de vías, entre otros. Estos factores también definirán el diseño específico de cada ciclovía (segregada, compartida, demarcada).

La energía necesaria para trasladarse entre cada par de estaciones se presenta en la tabla 5. Las celdas con borde negro indican un valor mayor al límite de $50 \mathrm{~kJ}$, lo que implica que no existe conectividad entre el par de estaciones correspondientes. Es importante observar que la matriz no es simétrica, ya que el valor de energía varía según el sentido del viaje. Por ejemplo, para llegar de la estación 7 a las 13 se requieren $11.8 \mathrm{~kJ}$, mientras que para el viaje en sentido opuesto se requieren $25.3 \mathrm{~kJ}$. A pesar de que los valores más bajos de consumo de energía se dan entre estaciones cercanas (valores ubicados cerca de la diagonal), hay excepciones donde el consumo de energía es elevado entre estaciones cercanas, debido a que entre ellas existen fuertes pendientes o calles con una 
Figura 5. Red ciclable para la conectividad del sistema de estaciones con equipamientos clave

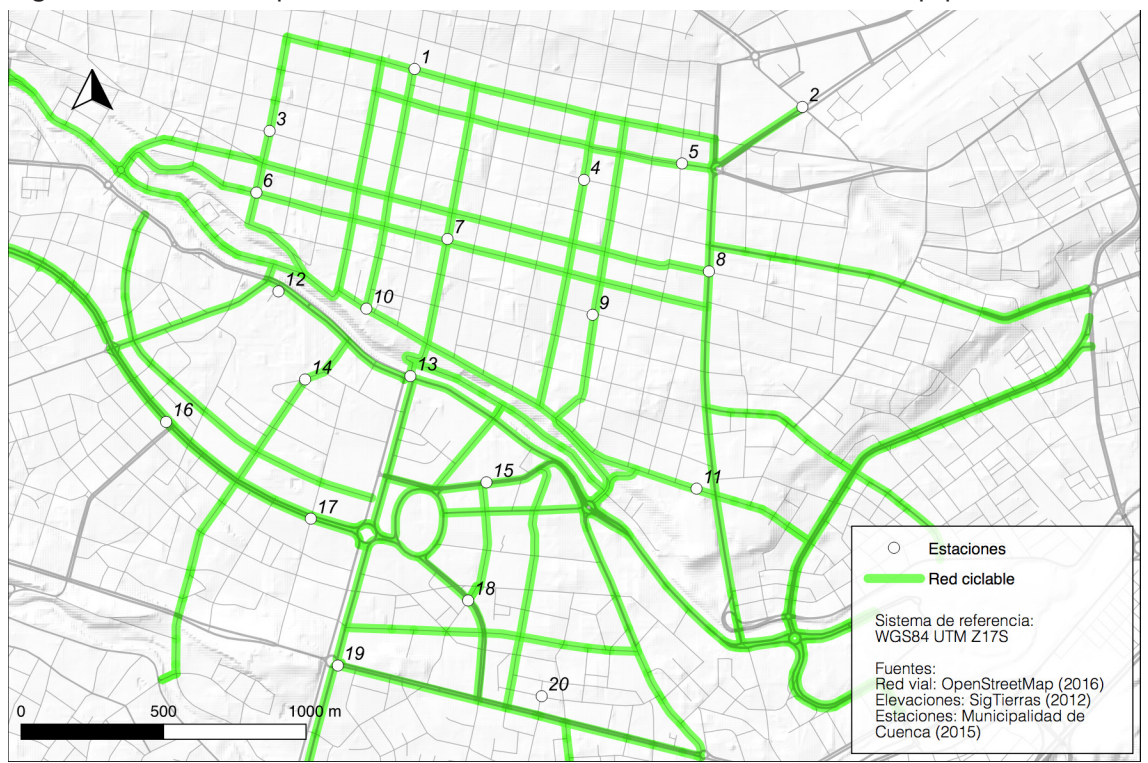

Fuente: elaboración propia a partir de OpenStreetMap (2016), MAGAP (2012) y Municipalidad de Cuenca (2015).

Tabla 5. Energía necesaria para pedalear entre estaciones. Las filas indican la estación de origen y las columnas, la estación de destino. Las celdas con borde negro muestran que no existe conectividad entre las estaciones debido a que se supera el límite de $50 \mathrm{~kJ}$

\begin{tabular}{|c|c|c|c|c|c|c|c|c|c|c|c|c|c|c|c|c|c|c|c|c|}
\hline & 1 & 2 & 3 & 4 & 5 & 6 & 7 & 8 & 9 & 10 & 11 & 12 & 13 & 14 & 15 & 16 & 17 & 18 & 19 & 20 \\
\hline 1 & & 18.5 & 16.0 & 0.7 & 12.1 & 18.6 & 6 & 16.9 & 17.6 & 11.4 & 26.7 & 21.4 & 21.2 & 24.0 & 28.7 & 30.6 & 32.3 & 31.4 & 38.2 & 38.7 \\
\hline 2 & 33.1 & & 45.9 & 14.4 & 4.8 & 47.6 & 30.8 & 7.7 & 18.6 & 39.5 & 17.5 & 50.4 & 44.8 & 52.5 & 36.1 & 63.9 & 50.2 & 37.9 & 55.9 & 38.6 \\
\hline 3 & 11.0 & 26.2 & & 14.9 & 19.3 & 2.8 & 10.9 & 20.1 & 19.1 & 11.9 & 29.9 & 13.5 & 19.3 & 20.7 & 26.8 & 22.8 & 25.5 & 29.5 & 36.3 & 36.3 \\
\hline 4 & 23.3 & 12.2 & 32.5 & & 5.4 & 33.5 & 16.6 & 7.6 & 7.9 & 25.3 & 17.3 & 36.3 & 28.9 & 36.6 & 26.3 & 45.5 & 35.5 & 30.4 & 41.2 & 31.0 \\
\hline 5 & 29.1 & 6.9 & 41.3 & 9.8 & & 43.0 & 26.2 & 5.0 & 14.0 & 34.9 & 14.8 & 45.8 & 38.4 & 46.1 & 33.4 & 55.1 & 47.6 & 35.3 & 53.2 & 35.9 \\
\hline 6 & 15.3 & 29.5 & 4.5 & 17.5 & 23.0 & & 8.5 & 20.8 & 16.3 & 9.1 & 25.0 & 10.7 & 16.5 & 17.9 & 24.0 & 20.0 & 22.6 & 26.7 & 33.5 & 33.5 \\
\hline & 15.3 & 21.5 & 21.5 & 9.5 & 15.0 & 17.3 & & 12.8 & 8.3 & 10.2 & 20.2 & 20.2 & 11.8 & 19.5 & 19.3 & 29.4 & 22.9 & 22.0 & 28.8 & 28.4 \\
\hline 8 & 34.2 & 10.0 & 42.4 & 12.7 & 5.4 & 41.4 & 24.5 & & 12.0 & 33.2 & 9.8 & 44.1 & 37.1 & 44.8 & 28.4 & 56.2 & 42.6 & 30.3 & 48.2 & 30.9 \\
\hline & 33.6 & 19.8 & 40.1 & 12.2 & 13.3 & 35.7 & 18.8 & 10.8 & & 24.8 & 12.3 & 36.0 & 26.9 & 34.6 & 21.8 & 45.2 & 33.5 & 23.7 & 39.2 & 24.3 \\
\hline 10 & 14.8 & 28.1 & 20.3 & 16.1 & 21.6 & 15.8 & 8.1 & 19.4 & 12.2 & & 15.9 & 11.7 & 8.9 & 16.7 & 16.4 & 20.9 & 20.0 & 19.1 & 25.9 & 26.4 \\
\hline & 49.6 & 25.4 & 57.8 & 28.1 & 20.8 & 51.2 & 35.2 & 15.4 & 19.5 & 35.4 & & 41.0 & 27.3 & 35.0 & 18.6 & 46.4 & 32.8 & 20.5 & 38.4 & 21.1 \\
\hline 12 & 33.1 & 47.4 & 30.3 & 35.4 & 40.9 & 25.8 & 26.4 & 38.7 & 31.8 & 20.1 & 29.8 & & 7.0 & 8.6 & 14.5 & 11.6 & 12.6 & 17.2 & 24.1 & 24.1 \\
\hline & 40.2 & 45.1 & 42.6 & 33.1 & 38.6 & 38.1 & 25.3 & 38.4 & 29.2 & 24.5 & 23.0 & 13.9 & & 7.9 & 7.5 & 25.0 & 11.4 & 10.2 & 17.0 & 17.0 \\
\hline 14 & 43.7 & 49.7 & 41.0 & 37.7 & 43.2 & 36.4 & 29.9 & 43.0 & 33.8 & 29.2 & 27.6 & 12.8 & 4.9 & & 9.5 & 12.7 & 5.7 & 12.3 & 19.1 & 19.2 \\
\hline 15 & 3.9 & 46.1 & 56.3 & 40.8 & 40.6 & 51.8 & 39.0 & 36.1 & 30.7 & 38.2 & 20.7 & 27.7 & 13.7 & 18.8 & & 28.4 & 14.8 & 5.7 & 20.4 & 11.2 \\
\hline 16 & 40.3 & 54.6 & 37.6 & 42.7 & 48.2 & 33.1 & 33.7 & 45.9 & 39.0 & 27.3 & 33.7 & 9.6 & 16.6 & 7.2 & 13.8 & & 5.5 & 12.8 & 18.4 & 19.6 \\
\hline $17 \mid$ & 1.0 & 53.4 & 48.2 & 43.9 & 47.9 & 43.7 & 36.1 & 43.4 & 38.0 & 35.4 & 28.0 & 19.1 & 10.9 & 8.1 & 8.0 & 13.4 & & 7.0 & 12.7 & 13.9 \\
\hline 18 & 8.8 & 50.6 & 61.3 & 45.6 & 45.1 & 56.8 & 44.0 & 40.6 & 35.5 & 43.2 & 25.2 & 32.6 & 18.7 & 23.9 & 7.0 & 28.7 & 15.1 & & 17.7 & 6.9 \\
\hline 19 & 4.6 & 55.8 & 57.0 & 47.5 & 50.3 & 52.5 & 39.7 & 45.8 & 41.6 & 39.0 & 30.4 & 28.4 & 14.5 & 19.6 & 11.6 & 24.4 & 10.8 & 6.3 & & 7.6 \\
\hline & 9.1 & 53.8 & 71.6 & 48.8 & 48.3 & 67.0 & 54.2 & 43.8 & 38.7 & 53.5 & 28.4 & 42.9 & 29.0 & 34.2 & 16.8 & 39.0 & 25.4 & 10.3 & 21.5 & \\
\hline
\end{tabular}

Energía (kilojulios)

\begin{tabular}{l|l|l|l|l}
$0-10$ & $10-20$ & $20-30$ & $30-40$ & $40-50$
\end{tabular}

Fuente: elaboración propia. 
Figura 6. Distribución óptima de diez estaciones de préstamo de bicicletas. Las líneas indican la asignación de equipamientos y servicios a la estación más cercana

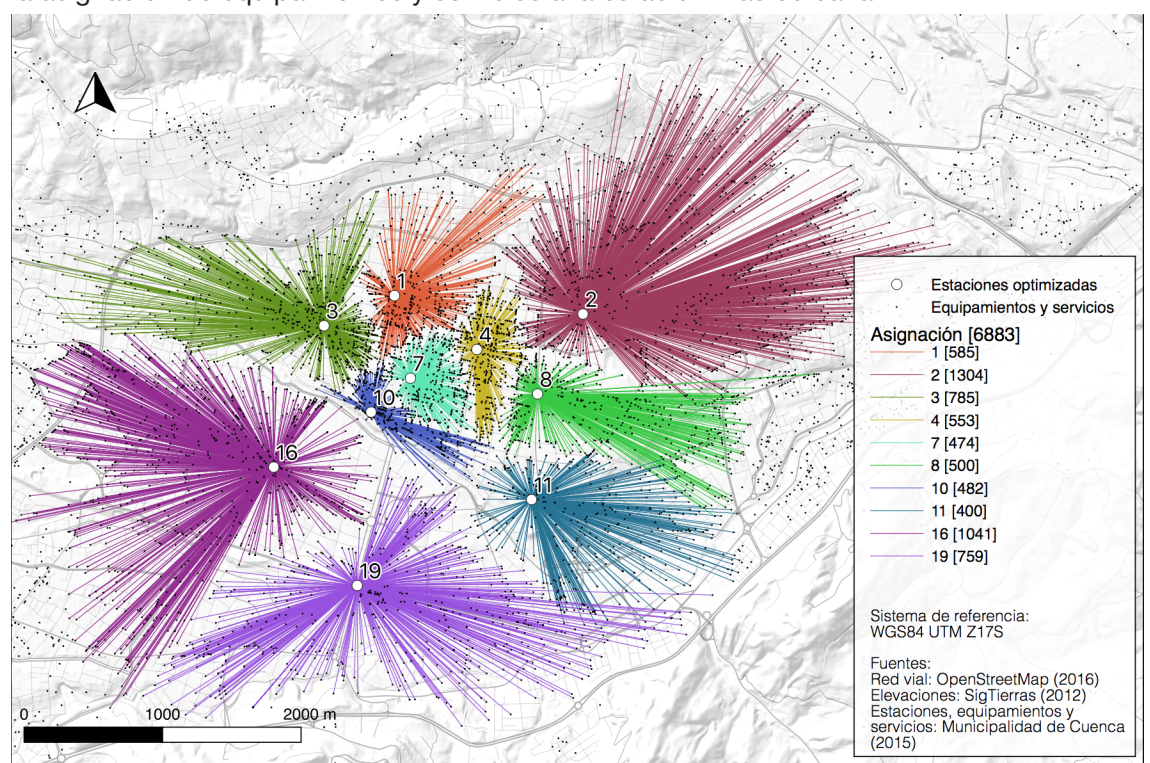

Fuente: elaboración propia a partir de OpenStreetMap (2016), MAGAP (2012) y Municipalidad de Cuenca (2015).

superficie muy rugosa (por ejemplo, de la estación 13 a la 9). Otra tendencia clara es la gran cantidad de energía requerida para viajar desde las estaciones ubicadas al sur (de la 17 a la 20) hacia aquellas ubicadas al norte (1, 2, 3, 6 y 7 ), las cuales están a una mayor altitud (esquina inferior izquierda de la matriz).

\subsection{Optimización de ubicaciones}

La figura 6 muestra la distribución óptima de diez estaciones utilizando el algoritmo de localización a partir de los valores de energía y los equipamientos y servicios asignados a cada una de ellas, en función del gasto mínimo de energía. En total, las diez estaciones darían cobertura a 6883 equipamientos dentro de sus respectivas áreas de influencia. Este análisis permite además estimar la demanda potencial de cada estación de préstamo de bicicletas. Se puede observar, por ejemplo, que las estaciones 2 y 16 tienen una alta demanda potencial, por lo que será necesario dotarlas de un mayor número de bicicletas.

\section{Conclusiones}

En este artículo hemos presentado una ampliación de un método de análisis de movilidad en bicicleta basado en el esfuerzo del pedaleo, incluyendo coeficientes de rodadura para diferentes tipos de superficie, además de la imple- 
mentación de una herramienta automatizada de análisis en un sistema de información geográfica que permite parametrizar el modelo para casos específicos y replicar el estudio en cualquier ciudad donde existan datos disponibles de elevación y de red vial de la plataforma libre OpenStreetMap. Adicionalmente, se ha demostrado la aplicabilidad del método para el análisis de una propuesta de un sistema de bicicleta pública en Cuenca (Ecuador), incluyendo el área de servicio del sistema, la conectividad de la red de ciclovías y la optimización de ubicaciones de las estaciones de préstamo.

Los resultados de este estudio resaltan la importancia de considerar el tipo de superficie de rodadura adicionalmente a la topografía y a las intersecciones en el cálculo de la energía consumida por el ciclista como valor de impedancia en los análisis de movilidad ciclística basados en la capacidad para realizar dicho trabajo. En el caso de estudio analizado se demostró que la omisión de este factor puede sobreestimar en promedio un $24 \%$ el área de servicio de la infraestructura ciclística.

El método propuesto ha sido implementado como una herramienta automatizada en el entorno ArcGIS, utilizando datos de red vial de la plataforma abierta OpenStreetMap y un modelo digital de elevaciones de libre acceso. Esto garantiza que el método puede ser aplicado en cualquier ciudad a nivel global donde existan este tipo de datos, con lo que se constituye en una herramienta útil para la planificación, el diseño y la evaluación de varios componentes de infraestructura ciclística urbana, tales como sistemas de préstamo de bicicletas y redes de ciclovías. Por ejemplo, un gobierno local puede utilizar la herramienta como apoyo para la decisión sobre el número y la ubicación óptimos de estaciones de préstamo de bicicletas y de talleres de mantenimiento y servicios. Así mismo, es posible estimar la intensidad de uso potencial de la red vial para decidir el diseño y la tipología de carriles de bicicleta exclusivos o compartidos.

El método propuesto también es útil para otros tipos de análisis que serán desarrollados en los siguientes pasos de nuestra investigación. Entre ellos están el análisis de accesibilidad combinando la bicicleta con el sistema de tranvía que actualmente se encuentra en construcción, lo cual es un elemento clave para un sistema intermodal (Griffin y Sener, 2016). Adicionalmente, se calculará la población servida por la infraestructura ciclística como insumo para la estimación de beneficios socioeconómicos y ambientales de las políticas de movilidad sostenible. Por otro lado, aunque el análisis está actualmente basado en el esfuerzo del pedaleo según las características físicas de la red vial, es posible conceptualizar la impedancia incluyendo otros factores que podrían afectar al nivel de ciclabilidad de las calles, tales como la intensidad de tráfico, la accidentabilidad, el ruido y la contaminación, así como valores perceptuales y estéticos de la ciudad. La inclusión de estos factores permitirá realizar una mejor estimación del potencial de uso ciclístico de la red vial, así como la modelación y evaluación de impacto de diferentes medidas y proyectos urbanos.

Aunque el presente estudio representa un avance sobre el método original, aún existen ciertas limitaciones que serán cubiertas en las siguientes fases de 
nuestra investigación. Por un lado, existen parámetros que afectan al consumo de energía por parte del individuo y que actualmente están asignados con valores únicos, tales como el peso del ciclista y la bicicleta o la velocidad del viento. Sin embargo, la herramienta desarrollada permite fácilmente personalizar estos valores según el usuario y en nuevas versiones posibilitará incluir datos geográficos acerca de la velocidad del viento para el análisis. Por otro lado, es necesario conocer cuán sensible es el método a los cambios en los parámetros de entrada, sea debido a la variación natural esperada o a posibles errores en las fuentes de información. Para esto se realizará un análisis de sensibilidad basado en simulaciones, lo cual permitirá definir los requerimientos de los parámetros de entrada desde un punto de vista pragmático. Finalmente, y no menos significativo, es importante lograr validar el método con datos reales, los cuales pueden ser obtenidos a partir de sensores colocados en las bicicletas o utilizados por los usuarios.

\section{Referencias bibliográficas}

BueHLER, Ralph y PuCher, John (2012). «Demand for Public Transport in Germany and the USA: An Analysis of Rider Characteristics». Transport Reviews, 32 (5), 541-567. <https://doi.org/10.1080/01441647.2012.707695>

De BaETS, Koen; De Mol, Johan y De MaeYer, Philippe (2011). «The use of digital maps for the evaluation and improvement of a bicycle-network and infrastructure». En: Colloquium Vervoersplanologisch Speurwerk 2011 (CVS - 2011). Stichting Colloquium Vervoersplanologisch Speurwerk (CVS). Recuperado de <https://biblio. ugent.be/publication/1955438>.

De NAZELLE, Audrey et al. (2011). «Improving health through policies that promote active travel: A review of evidence to support integrated health impact assessment». Environment International, 37 (4), 766-777. $<$ https://doi.org/10.1016/j.envint.2011.02.003>

ESRI (2015). ArcGIS 10.2. Redlands.

Fishman, Elliot; WaSHingTON, Simon y HaworTH, Narelle (2013). «Bike Share: A Synthesis of the Literature». Transport Reviews, 33 (2), 148-165. <https://doi.org/10.1080/01441647.2013.775612>

FRASER, Simon D. S. y LOCK, Karen (2011). «Cycling for transport and public health: A systematic review of the effect of the environment on cycling». European Journal of Public Health, 21 (6), 738-743. <https://doi.org/10.1093/eurpub/ckq145>

GEHL, Jan (2014). Ciudades para la gente. Buenos Aires: Infinito.

GREENSTEIN, Ashley Sarah (2015). Mapping bikeability: A spatial analysis on current and potential bikeability in Austin, Texas. Universidad de Texas en Austin. Recuperado de $<$ https://repositories.lib.utexas.edu/handle/2152/32204>.

Griffin, Greg Phillip y SEnER, Ipek Nese (2016). «Planning for Bike Share Connectivity to Rail Transit». Journal of Public Transportation, 19 (2), 1-22. <https://doi.org/10.5038/2375-0901.19.2.1>

Hanson, Susan (2004). «The context of urban travel». En: Hanson, Susan y GiULIANO, Genevieve (eds.). The geography of urban transportation. Nueva York: The Gildgford Press, 3-29. 
Hanson, Bailey y SeEger, Christopher J. (2014). ArcGIS Editor for OpenStreetMap. Iowa State University Extension and Outreach. Recuperado de <http://lib. dr.iastate.edu/extension_pubs/147/>.

Hidalgo, Dario y Huizenga, Cornie (2013). «Implementation of sustainable urban transport in Latin America». Research in Transportation Economics, 40 (1), 66-77. <https://doi.org/10.1016/j.retrec.2012.06.034>

Hölzel, Christin; Höchtl, Franz y Senner, Veit (2012). "Cycling comfort on different road surfaces». Procedia Engineering, 34 (suplemento C), 479-484. <https://doi.org/10.1016/j.proeng.2012.04.082>

INEC (2010). Información censal. Recuperado de <http://www.ecuadorencifras.gob. ec/institucional/home/> [Fecha de consulta: 01/05/2017].

Institute for Transportation And Development Policy (2013). The bikeshare planning guide. Recuperado de <https://www.itdp.org/wp-content/ uploads/2014/07/ITDP_Bike_Share_Planning_Guide.pdf $>$.

IsEKI, Hiroyuki y TingSTROM, Matthew (2014). "A new approach for bikeshed analysis with consideration of topography, street connectivity, and energy consumption». Computers, Environment and Urban Systems, 48, 166-177. $<$ https://doi.org/10.1016/j.compenvurbsys.2014.07.008>

Larsen, Jacob; Patterson, Zachary y El-Geneidy, Ahmed (2013). «Build It. But Where?: The Use of Geographic Information Systems in Identifying Locations for New Cycling Infrastructure». International Journal of Sustainable Transportation, 7 (4), 299-317. <https://doi.org/10.1080/15568318.2011.631098>

LIU, Suxia y ZHU, Xuan (2004). «Accessibility Analyst: An Integrated GIS Tool for Accessibility Analysis in Urban Transportation Planning». Environment and planning. B, Planning \& Design, 31 (1), 105-124.

$<$ https://doi.org/10.1068/b305>

Lugo, Adonia E. (2013). «CicLAvia and human infrastructure in Los Angeles: Ethnographic experiments in equitable bike planning». Journal of Transport Geography, 30, 202-207. <https://doi.org/10.1016/j.jtrangeo.2013.04.010>

MAGAP (2012). Programa SIGTierras. Recuperado de <http://www.sigtierras.gob.ec/> [Fecha de consulta: 01/05/2017].

Municipalidad de Cuenca (2015). Plan Operativo de Bicicletas. I. Municipalidad de Cuenca. Recuperado de <http://www.cuenca.gob.ec/?q=content/plan-de-movilidad>.

Olaya, Víctor (2014). Sistemas de Información Geográfica. Madrid: CreateSpace Independent Publishing Platform, 854. Recuperado de <http://volaya.github. io/libro-sig/>.

OpenStreetMap (2016). OpenStreetMap. Recuperado de <http://www.openstreetmap.org/> [Fecha de consulta: 01/05/2017].

Sener, Ipek; Eluru, Naveen y BHAT, Chandra (2009). "An Analysis of Bicyclists and Bicycling Characteristics: Who, Why, and How Much are they Bicycling?». En: Transportation Research Board (TRB) Annual Meeting. Washington, D. C. Recuperado de <http://www.ce.utexas.edu/prof/bhat/ABSTRACTS/sener_eluru_ bhat_bicycle_rev_Jan18_TRBstyle.pdf>.

ShaheEN, Susan; GuZman, Stacey y ZHANG, Hua (2010). «Bikesharing in Europe, the Americas, and Asia: Past, Present, and Future». Transportation Research Record: Journal of the Transportation Research Board, 2143, 159-167. <https://doi.org/10.3141/2143-20> 
TORRES, Andrea; SARMiEnto, Olga L.; STAUBER, Christine y Zarama, Roberto (2013). «The Ciclovia and Cicloruta Programs: Promising Interventions to Promote Physical Activity and Social Capital in Bogota». American Journal of Public Health, 103 (2), 23-30. <https://doi.org/10.2105/AJPH.2012.301142>

Wilson, David Gordon y Papadopoulos, Jim (2004). Bicycling Science. Cambridge, MA: MIT Press.

Winters, Meghan; Brauer, Michael; Setton, Eleanor M. y Teschke, Kay (2010). «Built environment influences on healthy transportation choices: Bicycling versus driving». Journal of Urban Health: Bulletin of the New York Academy of Medicine, 87 (6), 969-993.

<https://doi.org/10.1007/s11524-010-9509-6>

Yamashita, Yaeko; Dantas, Andre; Taco, Pastor y Yamamoto, Koshi (1999). "Geographical information system \& remote sensing in bicycle planning». Planning Review, 16, 327-332.

<https://doi.org/10.2208/journalip.16.327> 